\title{
QUALIDADE DA ASSISTÊNCIA OBSTÉTRICA RELACIONADA AO PARTO POR VIA VAGINAL: ESTUDO TRANSVERSAL
}

QUALITY OF OBSTETRIC ASSISTANCE RELATED TO VAGINAL DELIVERY: A CROSS-SECTIONAL STUDY

CALIDAD DE LA ASISTENCIA OBSTÉTRICA RELACIONADA AL PARTO VIA VAGINAL: ESTUDIO TRANSVERSAL

Rejane Sousa Romão ${ }^{1}$, Marceila de Andrade Fuzissaki ${ }^{2}$, Patrícia Santos Prudêncio ${ }^{3}$, Efigênia Aparecida Maciel de Freitas ${ }^{4}$.

\begin{abstract}
RESUMO
Objetivo: Identificar a qualidade da assistência durante o parto normal, segundo escore Bologna. A necessidade de avaliação da qualidade da assistência obstétrica visa atender às recomendações da Organização Mundial da Saúde e às diretrizes nacionais de assistência ao parto normal. Método: Trata-se de um estudo descritivo, de abordagem quantitativa, com delineamento transversal, realizado em um hospital. Participaram do estudo 82 puérperas internadas no Alojamento Conjunto. Para a coleta de dados foi adotada a técnica da entrevista semiestruturada e análise de prontuários. Para a análise dos dados foi utilizado o programa SPSS versão 20.0 e realizada a análise estatística pelo escore de Bologna. Resultados: As maiores frequências identificadas apresentaram pontuação 3 e 4, caracterizando a qualidade da assistência durante o parto normal do referido hospital como de média qualidade. Baixo índice de resultados iguais a 0 ou 1 que reportam ao esforço institucional em prol da melhora da assistência. Conclusão: Apesar dos achados positivos relacionados à garantia do direito ao acompanhante, utilização do partograma e predomínio da prática do contato pele a pele entre a mãe e o recém-nascido, há necessidade de a instituição adotar estratégias que visem a melhora da qualidade assistencial obstétrica.
\end{abstract}

Descritores: Parto humanizado; Qualidade da assistência à saúde; Enfermagem obstétrica; Saúde da mulher; Recém-nascido.

\section{ABSTRACT}

Objective: To identify the quality of care during normal delivery according to Bologna score. The need to assess the quality of obstetric care aims to meet the recommendations of the World Health Organization and the national guidelines for normal childbirth care. Method: This is a descriptive, quantitative, cross-sectional study conducted in a hospital. Eighty-two postpartum women admitted in the Rooming-in participated in the study. For the data collection, we adopted the semi-structured interview technique and analysis of medical charts. For the data analysis, the SPSS program version 20.0 was used and the statistical analysis was performed through the Bologna score. Results: The highest frequencies presented scores 3 and 4, characterizing the quality of care provided during the normal delivery of the referred hospital as of medium quality. The low index of results equal to 0 or 1 refer to the institutional effort for the care improvement. Conclusion: In spite of the positive findings related to the right to the companion, use of the partograph and predominance of the skin-to-skin contact between the mother and the newborn, the institution needs to adopt strategies aimed at improving the obstetric care quality.

Descriptors: Humanized delivery; Health care quality; Obstetric nursing; Women's Health; Newborn.

\section{RESUMEN}

Objetivo: Identificar la evaluación de la calidad de la asistencia durante el parto normal según escora Bologna. La necesidad de evaluar la calidad de la asistencia obstétrica tiene por objeto atender las recomendaciones de la Organización Mundial de la Salud y las directrices nacionales de asistencia al parto normal. Método: Se trata de un estudio descriptivo, de abordaje cuantitativo, con delineamiento transversal, realizado en un hospital. Participaron del estudio 82 puérperas internadas en el Alojamiento Conjunto. Para la recolección de datos fue adoptada la técnica de la entrevista semiestructurada y análisis de prontuarios. Para el análisis de los datos se utilizó el programa SPSS versión 20.0 y realizado el análisis estadístico por la puntuación de Bolonia. Resultados: Las mayores frecuencias identificadas presentaron puntuación 3 y 4 , caracterizando la calidad de la asistencia durante el parto normal del referido hospital como de media calidad. Bajo índice de resultados iguales a 0 o 1 que reporta al esfuerzo institucional en favor de la mejora de la asistencia. Conclusión: A pesar de los puntos positivos relacionados con la garantía del derecho al acompañante, utilización del partograma y predominio de la práctica del contacto piel a piel entre la madre y el recién nacido, hay necesidad de la institución adoptar estrategias que apunten a la mejora de la calidad asistencial obstétrica.

Descriptores: Parto humanizado; Calidad de la atención de salud; Enfermería obstétrica; Salud de la mujer; Recién nacido.

${ }^{1}$ Graduada em Enfermagem. Residente em Saúde da Criança pela Universidade Federal de Uberlândia. ${ }^{2}$ Graduada em Enfermagem. Doutora em Ciências da Saúde pela Universidade Federal de Uberlândia. ${ }^{3}$ Graduada em Enfermagem. Doutora em Enfermagem pela Escola de Enfermagem - USP/Ribeirão Preto. ${ }^{4}$ Graduada em Enfermagem. Doutora em Enfermagem Psiquiátrica pela Universidade de São Paulo. Docente da Universidade Federal de Uberlândia.

Como citar este artigo:

Romão RS, Fuzissaki MA, Prudêncio OS, et al. Qualidade da Assistência Obstétrica Relacionada ao Parto Por Via Vaginal: Estudo Transversal. Revista de Enfermagem do Centro Oeste Mineiro. 2018;8:e2907. [Access ]; Available in: DOI: http://dx.doi.org/10.19175/recom.v8i0.2907 


\section{INTRODUÇÃO}

A gestação e o parto são momentos que desencadeiam profundas mudanças na vida da mulher, casal e da família, e que merece uma atenção especial por parte dos profissionais de saúde. Nesse momento, há necessidade da implementação de uma assistência humanizada, que envolva o acolhimento e garanta um diálogo franco, livre de julgamentos e preconceitos. Uma assistência acolhedora e que proporcione a escuta ativa, possibilitando que a gestante e o parceiro expressem seus anseios, dúvidas, angústias, preocupações, no intuito de garantir uma atenção resolutiva e articulada com outros serviços de saúde, com o objetivo de garantir a continuidade da assistência ${ }^{(1)}$.

Em 2000, o Ministério da Saúde (MS) instituiu o Programa de Humanização no Prénatal e Nascimento (PHPN) visando à adoção de medidas que assegurassem melhorias no acompanhamento pré-natal e uma assistência ao parto, puerpério e neonatal de qualidade. 0 programa estabeleceu o direito ao atendimento digno e humanizado à mulher, seus familiares e ao recém-nascido. 0 programa teve, por base, o guia prático de atenção ao parto normal, desenvolvido pela Organização Mundial da Saúde (OMS) em 1996. Esse guia, baseado em evidências científicas, estimulava as equipes de saúde a realizarem procedimentos comprovadamente benéficos para a mulher e o bebê, evitando as intervenções desnecessárias ${ }^{(2)}$.

Um aspecto importante das ações humanizadas durante a evolução do parto é que as mesmas contribuem para a qualidade da assistência obstétrica, proporcionando, consequentemente, a segurança e o bem-estar materno-infantil, o que atende, assim, às recomendações da Organização Mundial da Saúde (OMS) $)^{(3)}$. Tais recomendações estabelecem a necessidade de cumprimento das metas relacionadas ao terceiro Objetivo de Desenvolvimento Sustentável (ODS) a serem alcançadas até 2030 e que tem como objetivo a implementação de ações e estratégias que visem assegurar a qualidade da saúde sexual e reprodutiva, reduzir a taxa de mortalidade materna global para menos de 70 mortes por 100.000 nascidos vivos, dentre outras ${ }^{(4)}$.

Desse modo, faz-se necessário avaliar o processo de cuidado, visando verificar se as práticas realizadas na atenção hospitalar estão em concordância com as evidências científicas disponíveis, assim como verificar o impacto de tais práticas na qualidade da assistência prestada durante o parto normal ${ }^{(5)}$.

Em 2000, partindo da premissa de que o parto é um evento fisiológico do corpo feminino, a OMS propôs, como estratégia para avaliar a qualidade dos serviços obstétricos prestados ao parto normal, um indicador denominado Escore de Bologna. Este instrumento é constituído por cinco medidas: presença de acompanhante durante o parto; presença de partograma; ausência de estimulação do trabalho de parto (uso de ocitocina, amniotomia, episiotomia, manobra de Kristeller) ou uso de instrumental (uso de fórceps e/ou vácuo extrator); parto em posição não supina; contato pele a pele da mãe com o recém-nascido (recomendado 30 minutos na primeira hora após o nascimento) ${ }^{(6)}$.

Outra estratégia de avaliação da qualidade da assistência prestada durante o parto normal foi estabelecida por meio da Portaria $n^{\circ} 353$, de 14 de fevereiro de $2017^{(7)}$, a qual aprovou as Diretrizes Nacionais de Assistência ao Parto Normal. Tais diretrizes possuem, como objetivo geral, sintetizar e avaliar sistematicamente a informação científica disponibilizada referente à prática assistencial ao parto e ao nascimento, no intuito de contribuir com a promoção, proteção e incentivo ao parto normal ${ }^{(8)}$. Para o cumprimento desse objetivo geral, algumas ações e estratégias são recomendadas na assistência ao parto normal, como: diminuição da variabilidade de condutas entre os profissionais no processo de assistência ao parto; redução de intervenções desnecessárias; implementação das práticas baseadas em evidências, dentre outras.

Tendo em vista que as ações humanizadas durante a assistência ao parto influenciam significativamente na qualidade da evolução do parto e na saúde do binômio, com consequente redução e otimização dos custos e redução nas taxas de mortalidade, o desenvolvimento do presente estudo é de fundamental importância, visto que propiciará uma reflexão acerca dos desafios e das potencialidades da implementação das recomendações estabelecidas pela $\mathrm{OMS}^{(3)}$, bem como as diretrizes Nacionais de Assistência ao Parto Normal $^{(8)}$, na prática clínica da assistência obstétrica. Destarte, o objetivo do presente estudo foi identificar a qualidade da assistência durante o parto normal, segundo escore Bologna. Para atender a esse objetivo, estabeleceu-se a seguinte questão norteadora: Qual a qualidade da assistência oferecida pelos profissionais de 
saúde durante o parto normal e quais os procedimentos realizados?

\section{MÉTODO}

Trata-se de um estudo descritivo, de abordagem quantitativa, transversal. Foi desenvolvido no setor de Alojamento Conjunto do Hospital de Clínicas de Uberlândia, pertencente à Universidade Federal de Uberlândia (HC-UFU), localizado no estado de Minas Gerais. A coleta de dados ocorreu entre janeiro a junho de 2017 e envolveu a participação de puérperas internadas no referido setor e que vivenciaram o parto por via vaginal.

Os critérios de inclusão estabelecidos foram: puérperas maiores de 18 anos, internadas no setor do alojamento conjunto do Sistema Único de Saúde (SUS), que tiveram o trabalho de parto e parto por via vaginal no ambiente hospitalar da instituição onde a pesquisa ocorreu. As puérperas que não atendiam aos critérios de inclusão foram excluídas. Além desses, foram estabelecidos como critérios de exclusão: as puérperas que, no momento da coleta de dados, relataram indisposição em participar do estudo, que se encontravam dormindo ou fora do leito, que se recusaram a participar da pesquisa, ou que tivessem passado por algumas intercorrências durante o parto e em casos de óbito fetal.

$\mathrm{O}$ cálculo amostral foi realizado utilizando o G*Power software, versão $3.1^{(9)}$. A amostra necessária de 82 participantes foi calculada baseada em teste exato (proporção) binomial, com tamanho de efeito esperado de 0,3 , um nível alfa de $0,05,99 \%$ de poder do teste.

Das 480 mulheres existentes no início do estudo, 82 compuseram a amostra final, conforme descrito na Figura 1.

Figura 1 - Processo de seleção das puérperas - Uberlândia (MG), Brasil, 2017.

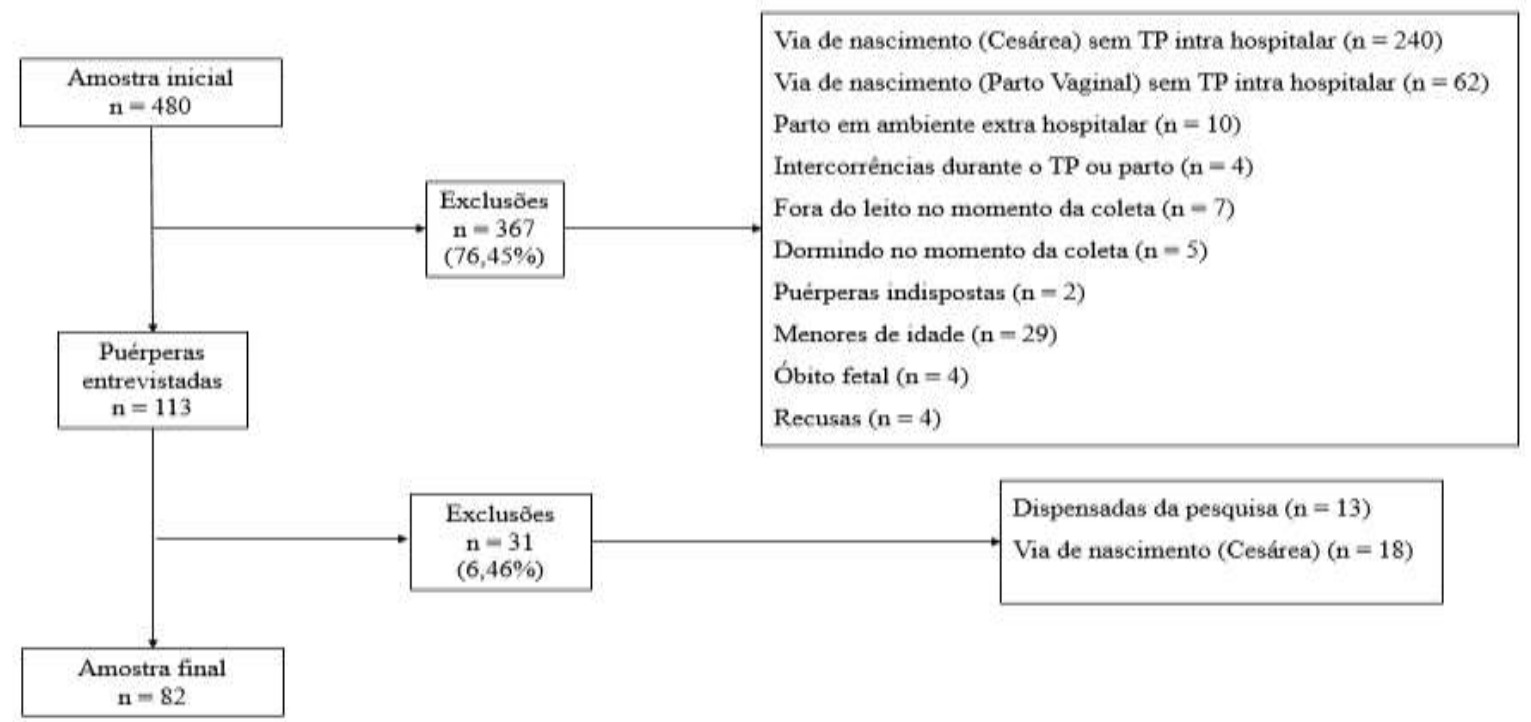

Fonte: Dados da pesquisa, 2018.

As participantes, selecionadas para participar do estudo, foram informadas e orientadas, individualmente, acerca dos objetivos, riscos e benefícios do presente estudo, bem como receberam o Termo de Consentimento Livre e Esclarecido (TCLE). A coleta de dados foi realizada pela pesquisadora principal deste estudo, por meio de entrevista semiestruturada e análise de prontuário. O local da coleta de dados ocorreu em ambiente privativo do setor de alojamento conjunto, no intuito de garantir a privacidade, sigilo das informações relatadas pelas puérperas, bem como evitar possíveis vieses da pesquisa.

A pesquisa foi submetida ao Comitê de Ética em Pesquisa (CEP), da Universidade Federal de Uberlândia (UFU), sendo aprovado em 06/12/2016 sob o número do parecer: 1.864.935. Para a organização dos dados, foi realizada a dupla digitação dos mesmos no Microsoft Excel Professional Plus 2016. Foi elaborado um dicionário de dados (Codebook) com a especificação de todas as variáveis abordadas no questionário aplicado e adotada a técnica de validação por dupla entrada (digitação) no intuito de garantir a fidelidade no momento da transcrição dos dados ${ }^{(10)}$. Após a validação por dupla digitação, realizou-se a análise descritiva das variáveis do estudo por meio da importação da planilha do Excel para o programa estatístico SPSS, versão 20.0 . 
As variáveis incluídas nas análises foram separadas em características sociodemográficas (idade, estado civil e escolaridade) relatadas pelas participantes; histórico de doenças anteriores à gestação que incluiu a presença de doenças crônicas e agudas (Síndrome da Imunodeficiência Adquirida, Diabetes Mellitus, Hipertensão Arterial, Hipercolesterolemia), as quais podem estar relacionadas à mortalidade neonatal, baixo peso ao nascer, prematuridade e ao surgimento de outras complicações obstétricas ${ }^{(11)}$, coletado do prontuário hospitalar; histórico obstétrico: paridade (número de partos ao qual a mulher se submeteu, categorizada entre multíparas e primíparas), aborto (definido como a interrupção da gestação até 22 semanas ou feto com peso menor de 500 gramas ou medindo menos de 16 $\mathrm{cm}$, caso se desconheça o período gestacional) ${ }^{(12)}$ e cesáreas anteriores; aspectos relacionados à gestação atual como a sua duração (expressa pela idade gestacional baseada na ultrassonografia realizada no primeiro trimestre ou na data da última menstruação, registrada em prontuário hospitalar), via de nascimento (distinção da ocorrência de parto via vaginal com o uso do fórceps ou não), escala de APGAR no primeiro e quinto minuto de vida da criança (escala de avaliação das funções físicas essenciais ao neonato nos primeiros minutos após o nascimento, que engloba uma pontuação de 0 a 2 para as características: frequência cardíaca, esforço respiratório, tônus muscular, irritabilidade reflexa e coloração da pele ${ }^{(13)}$, sendo coletado do prontuário médico.
Para avaliar a qualidade da assistência durante o trabalho de parto e parto, considerouse o escore de Bologna. Esse instrumento de avaliação é constituído por 5 medidas: 1) presença de um acompanhante no parto; 2 ) uso do partograma; 3) ausência de estimulação do trabalho de parto; 4) parto na posição não supina; e 5) contato pele a pele entre mãe e filho. A cada variável é atribuída a nota " 1 ", se presente, e " 0 ", se ausente. $O$ resultado final é obtido com a somatória das pontuações de todas as medidas. A pontuação máxima " 5 " corresponde à maior qualidade de assistência, e a pontuação " 0 " corresponde a um parto mal conduzido. Pontuações entre esses limites correspondem às variações da qualidade de assistência $^{(5-6)}$. Dentre os estímulos analisados, considerou-se a amniotomia, a episiotomia, o uso de medicamentos estimulantes (ocitocina e misoprostol) e o uso de fórceps durante o período expulsivo. Ressalta-se que os dados perdidos foram categorizados como "não identificado" e não ultrapassaram a taxa aceita que é de até $5 \%$ do total de dados de uma base de dados.

\section{RESULTADOS E DISCUSSÃO}

O presente estudo contou com a participação de 82 puérperas com idade média de 25,3 anos, que deram entrada no Pronto Socorro do Hospital das Clínicas de Uberlândia da Universidade Federal de Uberlândia (HC-UFU) e que foram assistidas durante o trabalho de parto e parto no Centro Obstétrico do referido hospital. Outras características sociodemográficas estão descritas na Tabela 1.

Tabela 1 - Características sociodemográficas das mulheres - Uberlândia (MG), Brasil, 2017.

\begin{tabular}{lcc}
\hline Características & Todas as puérperas \\
Idade & $\mathrm{n}(82)$ & $100(\%)$ \\
$18-24$ anos & 43 & 52,4 \\
$25-41$ anos & 39 & 47,6 \\
Estado Civil & & 64,6 \\
Solteira & 53 & 35,4 \\
Casada & 29 & 43,9 \\
Escolaridade & & 20,7 \\
Médio Completo & 36 & 11 \\
Médio Incompleto & 17 & 11 \\
Fundamental Incompleto & 9 & 9,7 \\
Fundamental Completo & 9 & 3,7 \\
Superior Incompleto & 8 & 3 \\
Superior Completo & 3 & \\
\hline
\end{tabular}

Fonte: Dados da pesquisa, 2018.

Em relação ao histórico de doenças anteriores, 81 (98,8\%) mulheres não possuíam enfermidades antes da gestação e $1(1,2 \%)$ estava com hipercolesterolemia. Outras características do histórico clínico obstétrico estão descritas na Tabela 2. 
Tabela 2 - Histórico obstétrico das mulheres - Uberlândia (MG), Brasil, 2017.

\begin{tabular}{|c|c|c|}
\hline \multirow{2}{*}{ Características } & \multicolumn{2}{|c|}{ Todas as mulheres } \\
\hline & $\mathrm{n}(82)$ & $100(\%)$ \\
\hline \multicolumn{3}{|l|}{ Paridade } \\
\hline Multípara & 55 & 67,1 \\
\hline Primípara & 27 & 32,9 \\
\hline \multicolumn{3}{|l|}{ Aborto anterior } \\
\hline Não & 63 & 76,8 \\
\hline Sim & 19 & 23,2 \\
\hline \multicolumn{3}{|l|}{ Cesárea anterior } \\
\hline Não & 69 & 84,1 \\
\hline Sim & 13 & 15,9 \\
\hline \multicolumn{3}{|l|}{ Duração da gestação } \\
\hline $37-40$ semanas & 72 & 87,8 \\
\hline 32-36 semanas & 6 & 7,3 \\
\hline 41 semanas & 3 & 3,7 \\
\hline Não identificado & 1 & 1,2 \\
\hline \multicolumn{3}{|l|}{ Via de Nascimento } \\
\hline Vaginal sem fórceps & 79 & 96,3 \\
\hline Vaginal com fórceps & 3 & 3,7 \\
\hline \multicolumn{3}{|c|}{ Escala de APGAR no 19 minuto de vida } \\
\hline Pontuação de 7 a 10 & 77 & 93,9 \\
\hline Não identificado & 3 & 3,7 \\
\hline Pontuação de 0 a 6 & 2 & 2,4 \\
\hline \multicolumn{3}{|c|}{ Escala de APGAR no 5o minuto de vida } \\
\hline Pontuação de 7 a 10 & 80 & 97,6 \\
\hline Não identificado & 2 & 2,4 \\
\hline
\end{tabular}

Fonte: Dados da pesquisa, 2018.

As condutas assistenciais ao parto, de acordo com o Escore de Bologna, estão descritas na Figura 2. Ressalta-se que, dentre os estímulos realizados, temse a amniotomia $29(35,4 \%)$, a episiotomia $23(28 \%)$; o uso da ocitocina $20(24,4 \%)$ e o misoprostol 12
$(14,6 \%)$ e o uso de fórceps durante o período expulsivo $3(3,6 \%)$. E dentre os familiares que se destacaram na escolha das puérperas para 0 acompanhamento, têm-se o pai da criança $(52,4 \%$, $n=43)$ e a mãe da parturiente $(19,5 \%, n=16)$.

Figura 2 - Padrões de Qualidade do Escore de Bologna para os partos da maternidade do Hospital de Clínicas de Uberlândia, (MG), Brasil, 2017.

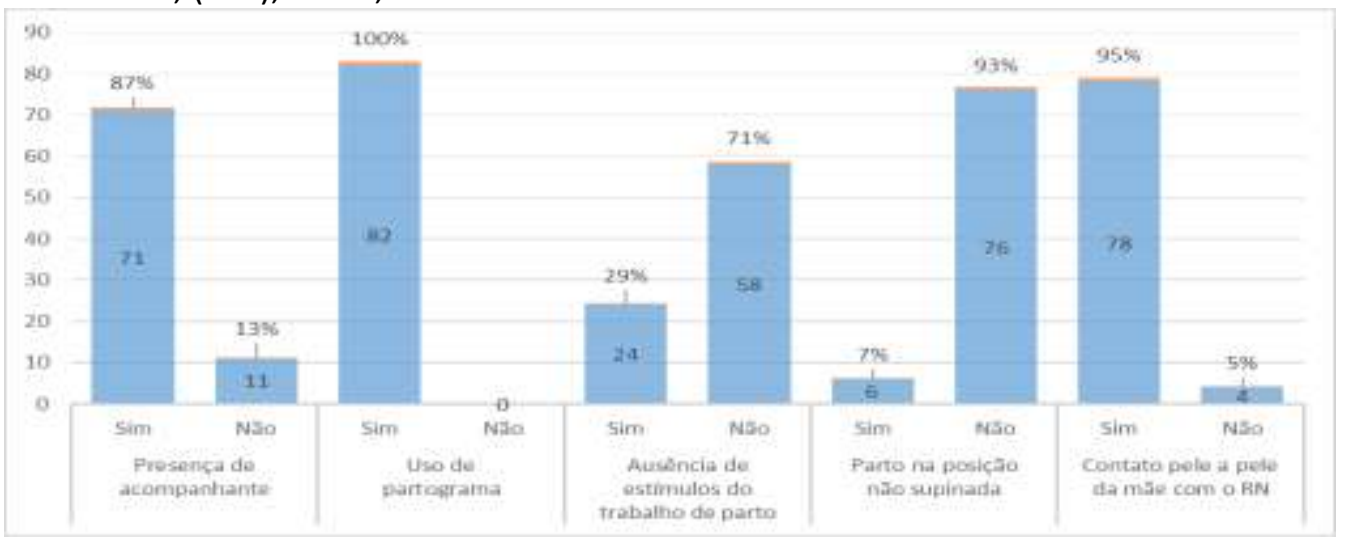

Fonte: Dados da pesquisa, 2018.

No que se refere à presença de acompanhante durante o trabalho de parto e parto, a maioria das mulheres do presente estudo responderam positivamente. Além disso, não foram relatados casos em que a ausência de acompanhante deveu-se à recusa do hospital em respeitar esse direito, previsto na Lei n. 011.108 de 2005, sendo um bom indicativo da qualidade de assistência do HC-UFU. Sabe-se que o acompanhante de escolha da parturiente transmite segurança a ela, melhora as chances de parto normal e de satisfação da mulher com o parto, além de diminuir o uso de analgesia farmacológica e riscos de complicações ${ }^{(14)}$.

O cumprimento do hospital HC-UFU em assegurar o direto ao acompanhante por parte da 
maioria das parturientes diverge do que foi encontrado na literatura ${ }^{(14-15)}$. Em pesquisa realizada com 160 gestantes e puérperas pertencentes a duas maternidades filantrópicas do Brasil, identificou-se que 92 (57,5\%) das participantes desconheciam a referida lei e que $106(66,2 \%)$ delas não dispunham da presença do acompanhante em todo $o$ processo de parturição ${ }^{(15)}$. Resultados referentes à pesquisa "Nascer no Brasil", realizada em 2012, com 266 hospitais e um total de 23.940 puérperas, também identificaram a presença contínua do acompanhante em apenas $18,8 \%$ dos casos, com picos de acompanhamento antes ou durante a admissão $(70,1 \%)$ e já no alojamento conjunto (61,3\%). Entretanto, a presença do acompanhante foi indicada por $84,5 \%$ das mulheres como um elemento importante para ter um parto melhor e mais calmo ${ }^{(14)}$.

Na condição de acompanhante, o pai pode ter o papel de apoiar a mãe, ajudando-a a passar pelas contrações e a realizar os métodos não farmacológicos de alivio da dor ou ao cortar o cordão umbilical. Além disso, estudos comprovam que a participação paterna durante o processo parturitivo é considerada como positiva pelas puérperas, uma vez que favorece o vínculo entre o casal e o recém-nascido ${ }^{(16)}$.

Tais evidências demonstram a importância de a participação do pai ser constantemente estimulada durante a gestação, parto, nascimento e puerpério. Além disso, vários estudos científicos comprovaram que as gestantes que tiveram seus parceiros como acompanhantes sentiram-se mais seguras e confiantes para a experiência do parto ${ }^{(1)}$. Diante de tais evidências, ressalta-se a importância da atuação dos profissionais de saúde na estimulação da participação paterna durante todo o processo parturitivo.

Outro indicador da qualidade da assistência é a utilização do partograma, observado em todos os prontuários. A utilização do partograma possibilita aos profissionais que assistem ao parto, identificar desvios do bem estar materno e fetal e da evolução do parto ${ }^{(6)}$, bem como identificar as mulheres que necessitam de uma intervenção obstétrica. Além da utilização do partograma pelos profissionais de saúde envolvidos na assistência a parturiente, um importante aspecto a ser periodicamente avaliado e que influencia na qualidade da assistência é o nível de conhecimento dos profissionais que preenchem o partograma, assim como realizado em um estudo na Etiópia que constatou que mais da metade dos participantes apresentaram um bom nível de conhecimento sobre o partograma, tendo sido recomendada a realização periódica de treinamento relacionado ao partograma ${ }^{(17)}$. No presente estudo, avaliou-se apenas a utilização do partograma, sendo constatada a necessidade de novos estudos que avaliem também o nível de conhecimento dos profissionais de saúde quanto ao seu preenchimento e análise para direcionamento da conduta clínica.

Quanto à utilização de estímulos ao trabalho de parto, a OMS recomenda que a prática da episiotomia não ultrapasse $10 \%$ dos casos, pois não previne a ocorrência de laceração perineal mais grave, e está relacionada à insatisfação materna e à dor no puerpério. Apesar de tal recomendação, no presente estudo, identicou-se que a prática da episiotomia atingiu $28 \%$. Nesse aspecto, ressalta-se a importância da conscientização e capacitação dos profissionais de saúde envolvidos na assistência direta ao parto quanto à necessidade de maiores esforços para atingir a meta estipulada pela OMS, garantindo assim a qualidade da assistência obstétrica. Além disso, as puérperas devem ser orientadas a buscarem informações sobre a episiotomia, pois o desconhecimento sobre essa prática é considerado um fator que contribui para a realização rotineira desse procedimento ${ }^{(18)}$.

Outro aspecto que deve ser banido das práticas rotineiras de assistência é o uso de uterotônicos, sendo benéficos apenas em casos comprovados de disfunção da contratilidade uterina, estando relacionados ao aumento do uso de fórceps, episiotomia, analgesia e realização de cesarianas de emergência ${ }^{(6)}$. Um estudo descritivo e retrospectivo realizado em uma maternidade pública, no município do Rio de Janeiro, revelou que, das parturientes não submetidas à episiotomia, 56,6\% ( $n=605)$ tiveram laceração perineal de 10 grau; $38,6 \% \quad(n=431)$ permaneceram com o períneo íntegro e apenas $4,6 \%(n=51)$ delas sofreram lacerações de 20 grau. Esses dados indicam que as lesões genitais no parto vaginal podem ser prevenidas através de uma boa assistência, uma vez que, de acordo com as evidências científicas, o uso rotineiro desse procedimento não reduz o risco de trauma perineal severo (lacerações de 3으 e 4음 grau), incontinência urinária de esforço e dispareunia, além de estar associado ao desconforto e dores perineais durante o puerpério ${ }^{(19)}$. 
A posição do parto, também avaliada pelo escore de Bologna, influencia na evolução de um parto com menos distócias. Avaliou-se, no presente estudo, a ocorrência de posição não supina durante parto, em apenas $7 \%$, ou seja, um valor muito aquém do esperado. Tal panorama demonstra a necessidade de capacitação dos profissionais de saúde das instituições hospitalares em orientarem as parturientes quanto aos benefícios e ao direito de adotar outras posições durante o momento do parto.

É preciso ressaltar que, qualquer posição não supina, que seja adotada, está associada à diminuição da duração do período expulsivo e da necessidade de se realizar episiotomia. Além disso, facilita o processo fisiológico do parto, pois relaxa a pelve, aumenta a liberdade de movimentos e a flexibilidade do quadril, os quais ajudam na rotação da apresentação fetal, além de diminuir as sensações dolorosas ${ }^{(19-20)}$.

Além disso, pontua-se que o posicionamento horizontal para o parto está relacionado a uma maior compressão da artéria aorta e veia cava, dificultando a troca gasosa entre o binômio mãe e filho e à menor efetividade das contrações uterinas ${ }^{(21)}$. Entretanto, a litotomia ainda é a posição mais utilizada para o nascimento em ambiente hospitalar, devido à maior facilidade para que os profissionais possam monitorar as contrações através da palpação abdominal, realizar exames vaginais e manobras invasivas, verificar a posição da cabeça fetal e a frequência cardíaca do bebêt $\hat{e}^{(22)}$.

Outra evidência que demonstra os benefícios do posicionamento materno durante $o$ trabalho de parto foi apresentada em um estudo realizado na Itália, que identificou que a adoção da posição de escolha da parturiente contribuiu para a redução da dor materna, do parto vaginal operatório, na cirurgia cesariana e nas taxas de episiotomia ${ }^{(22)}$. Tais achados refletem a importância da atuação dos profissionais de saúde no encorajamento do protagonismo materno durante o trabalho de parto, envolvendo a decisão pelo posicionamento mais confortável.

Em relação à prática do contato pele a pele entre a mãe e o recém-nascido, o mesmo ocorreu em $95 \%$ dos casos, ou seja, um ponto positivo do atendimento proporcionado às parturientes pelo HC-UFU, uma vez que é de conhecimento que, nos primeiros minutos de vida, os bebês devem ser colocados junto à mãe de forma contínua, por pelo menos meia hora, com o objetivo de promover o reconhecimento do bebê e estimular o aleitamento materno ${ }^{(23)}$. Esse contato beneficia o aumento da quantidade de alguns hormônios que estimula a criação do vínculo mãe-filho e o reflexo da descida do leite. A amamentação, na primeira hora de vida, por sua vez, garante a proteção imunológica precoce do bebê e o fornecimento de alimento rico em nutrientes essenciais ${ }^{(24)}$.

Além disso, o contato pele a pele, no período imediato após o nascimento, fornece proteção e calor para o recém-nascido, que sofreu uma diminuição na temperatura corporal devido à saída do útero materno para o ambiente externo ${ }^{(24)}$. Nesse contexto, com o propósito de identificar a prevalência do cumprimento dessa prática, um estudo realizado no Nordeste brasileiro com 107 puérperas demonstrou que tal prática foi comprometida quando associada à cirurgia cesárea ${ }^{(23)}$. Outros fatores de risco identificados em um estudo de revisão sistemática foram: a baixa renda familiar, idade materna menor que 25 anos, baixa escolaridade materna, ausência de consultas pré-natais, parto domiciliar, falta de orientação sobre amamentação no pré-natal e a prematuridade ${ }^{(25)}$.

Evidencia-se, portanto, a necessidade de considerar tais fatores de risco durante a assistência. Além disso, outro aspecto que deve ser analisado em estudos posteriores é como esse contato pele a pele tem sido realizado, sua duração e sua efetividade sobre a amamentação.

As maiores frequências encontradas neste estudo para o Escore de Bologna foram de pontuações 3 e 4 , sugerindo que a assistência obstétrica do HC-UFU é de média qualidade. Porém, o baixo índice de resultados iguais a 0 ou 1 sugere que a instituição tem realizado esforços para se adequar à utilização de práticas mais benéficas do atendimento ao parto (Figura 3). 
Figura 3 - Pontuação do Escore de Bologna para os atendimentos realizados na maternidade do Hospital de Clínicas de Uberlândia, (MG), Brasil, 2017.

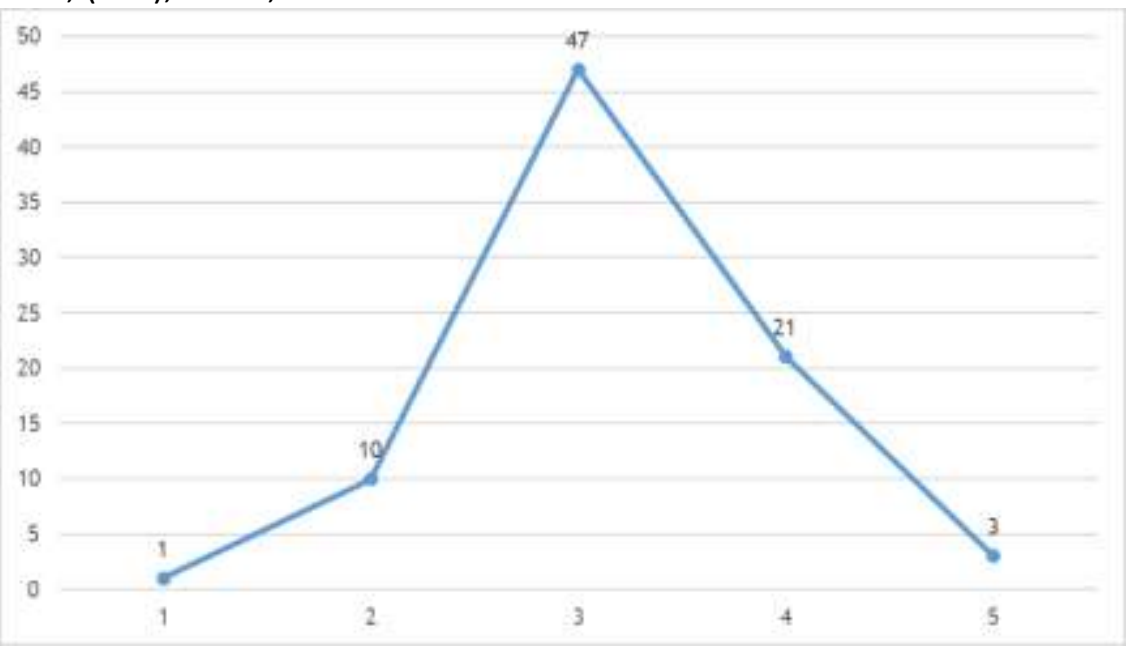

Fonte: Dados da pesquisa, 2018.

Em um estudo, que avaliou três maternidades em Natal no Rio Grande do Sul, com uma amostra de 314 puérperas, identificouse um índice geral de Bologna médio de 2,1; o principal tipo de estimulação no trabalho de parto utilizado foi a ocitocina $(52,9 \%)$ e os percentuais mais baixos encontrados referem-se ao parto na posição não supina $(0,3 \%)$ e uso do partograma $(2,2 \%)^{(5)}$. Outro estudo realizado no Município de Curitiba-PR, em três instituições do SUS, também identificou índices baixos. A maternidade $A$ obteve escores finais de 1 (78,7\%) e $2(21,3 \%)$ para os 80 prontuários pesquisados. Já a instituição $\mathrm{B}$, dentre os 126 prontuários observados, os escores finais $1(43,7 \%)$ e 2 $(44,4 \%)$ foram os mais prevalentes. A instituição C obteve escores finais de 1 (30,5\%), 2 (45,5\%) e 3 (19\%) para os 200 prontuários analisados ${ }^{(6)}$.

Diante do exposto, evidencia-se que o principal achado do estudo foi que o hospital ofereceu uma boa qualidade de assistência, com a maioria das mulheres tendo a presença de um acompanhante de escolha durante o parto, além da existência de contato pele a pele entre mãe e recém-nascido. Entretanto, condutas como a adoção de posição supina e a utilização de algum método de estimulação ao trabalho de parto foram extensivamente identificadas. Isso indica a necessidade de melhoria de alguns aspectos da assistência no intuito de atender às diretrizes nacionais da assistência ao parto normal, bem como colaborar para o cumprimento das metas do terceiro ODS proposto pela OMS.

Dentre as limitações do estudo, destacase o fato de a amostra ter sido homogênea. Nesse sentido, sugere-se, em pesquisas futuras, a inclusão de uma amostra mais heterogênea, considerando outro tipo de via de nascimento e a inclusão da saúde suplementar. Isso possibilitará identificar a associação desses fatores com a qualidade da assistência, contribuindo, assim, para a prática profissional em diversos cenários.

\section{CONCLUSÃO}

Os achados deste estudo demonstram que as condutas recomendadas pela OMS para o parto e nascimento, como contato pele a pele com duração superior a 30 minutos, posições não supinas para o parto, a utilização de episiotomia e uterotônicos apenas em casos que, comprovadamente, necessitem dessas intervenções, devem ser periodicamente avaliadas pela gerência hospitalar, uma vez que a análise da qualidade assistencial pelo escore de Bologna demonstrou aspectos que demandam melhorias.

Entretanto, percebeu-se que a presente instituição conseguiu trabalhar, de maneira efetiva, alguns aspectos da humanização da assistência obstétrica, como a garantia do direito ao acompanhante, utilização do partograma para todas as participantes da pesquisa e o predomínio da prática do contato pele a pele entre a mãe e o recém-nascido. Tais ações devem ser incentivas continuamente pelas instituições de saúde, visando uma melhor qualidade da assistência obstétrica e contribuindo para o bemestar e saúde materna e neonatal e, também, para a redução de custos que, indiretamente, estão relacionados à não realização dessas práticas. 
Por fim, outra estratégia é investir na capacitação dos profissionais de saúde que compõem a equipe multidisciplinar de assistência ao parto, valorizando os saberes de cada membro em sua especialidade, e dividindo as funções de maneira mais homogênea.

\section{REFERÊNCIAS}

1. Ministério da Saúde. Secretaria de Atenção à Saúde. Orientações básicas de atenção integral à saúde de adolescentes nas escolas e unidades básicas de saúde. Brasília: Ministério da Saúde; 2013.

2. Silva TC, Bisognin $P$, Prates $L A$, Wilhelm LA, Bortoli CFC, Ressel LB. As boas práticas de atenção ao parto e nascimento sob a ótica de enfermeiros. Biblioteca Lascasas 2016 [citado em 7 out 2018]; 12(1):1-22. Disponível em: http://www.indexf.com/lascasas/documentos/lc0 886.php

3. Organização das Nações Unidas (ONU). Documentos temáticos: Objetivos de desenvolvimento sustentável 1,2,3,5,9,14. Brasília: ONU; 2017.

4. Secretaria de Governo da Presidência da República. Ministério do Planejamento, Desenvolvimento e Gestão. Relatório nacional voluntário sobre os objetivos de desenvolvimento sustentável: Brasil 2017. Brasília: Presidência da República; 2017.

5. Carvalho IS, Brito RS. Utilização do índice de Bologna para avaliação da assistência ao parto normal. Rev Esc Enferm USP. 2016;50(5):742-9. DOI: $10.1590 /$ S0080-623420160000600005

6. Oliveira FAM, Leal GCG, Wolff LDG, Gonçalves LS. O uso do Escore de Bologna na avaliação da assistência a partos normais em maternidades. Rev Gaúcha Enferm. [online] 2015; 36:177-184. DOI: 10.1590/1983-1447.2015. esp. 56940

7. Brasil. Secretaria de Atenção à Saúde da Receita Federal. Portaria no 353, 14 de fevereiro de 2017. Diário Oficial da União 2017;36:37.

8. Comissão Nacional de Incorporação de Tecnologias do SUS (CONITEC). Diretrizes Nacionais de assistência ao parto normal. Brasília: CONITEC; 2017.

9. Faul F, Erdfelder E, Lang AG, Buchner A. G* Power 3: A flexible statistical power analysis program for the social, behavioral, and biomedical sciences. Behav Res Methods 2007 [citado em 7 out 2018]; 39(2):175-91. Disponível em: https://www.ncbi.nlm.nih.gov/pubmed/1769 $\underline{5343}$
10. Hulley SB, Cummings SR, Browner WS, Grady DG, Newman TB. Delineando a pesquisa clínica. 4a ed. Porto Alegre: Artmed; 2015.

11. Moura LAM, Alencar GP, Silva ZP, Almeida MF. Internações por complicações obstétricas na gestação e desfechos maternos e perinatais, em uma coorte de gestantes no Sistema Único de Saúde no Município de São Paulo, Brasil. Cad Saúde Pública 2018;34(1):e00188016. DOI: 10.1590/0102-311x00188016

12. Brasil. Ministério da Saúde. Parto, aborto e puerpério: Assistência humanizada à mulher. Brasília: Secretaria de Políticas de Saúde, Área Técnica da Saúde da Mulher; 2001.

13. Brasil. Ministério da Saúde. Secretaria de Atenção à Saúde. Atenção à saúde do recémnascido: Guia para os profissionais de saúde. 2a ed. atual. Brasília: Ministério da Saúde; 2014.

14. Diniz CSG, D'Orsi $E$, Domingues RMSM, Torres JA, Dias MAB, Schnek CA, et al. Implementação da presença de acompanhantes durante a internação para o parto: Dados da pesquisa nacional Nascer no Brasil. Cad Saúde Pública.2014;30(supl):140-153. DOI: 10.1590/01 02-311X00127013

15. Santos ECP, Lima MR, Conceição LL, Tavares CS, Guimarães AMAN. Conhecimento e aplicação do direito do acompanhante na gestação e parto. Enferm Foco 2016;7(3/4):61-5. DOI: 10.21675/ 2357-707X.2016.v7.n3/4.918

16. Holanda SM, Castro RCMB, Aquin PS, Pinheiro AKB, Lopes LG, Martins ES. Influência da participação do companheiro no pré-natal: Satisfação de primíparas quanto ao apoio no parto. Texto Contexto-Enferm. 2018; 27(2):e3800016. DOI: 10.1590/0104-07072018 0003800016

17. Markos D, Bogale D. Knowledge and utilization of partograph among health care professionals in public health institutions of Bale zone, Southeast Ethiopia. Public Health. 2016;137:162-8. DOI: 10.1016/j.puhe.2016.02. $\underline{026}$

18. Pompeu KC, Scarton J, Cremonese L, Flores RG, Landerdahl MC, Ressel LB. Prática da episiotomia no parto: Desafios para a enfermagem. Rev Enferm Cent-Oeste Min. 2017;7(1):1-8. DOI: 10.19175/recom.v7i0.1142

19. Silva TG, Costa GAB, Pereira ALF. Cuidados de enfermagem obstétrica no parto normal. Cogitare Enferm. 2011 [citado em 7 out 2018]; 16 (1):82-7. Available in: https://revistas.ufpr.br/ cogitare/article/view/21116 
20. Giglio MRP, França E, Lamounier JA. Avaliação da qualidade de assistência ao parto normal. Rev Bras Ginecol Obstet. 2011; 33(10):297-304. DOI: 10.1590/S0100-72032011 $\underline{001000005}$

21. Leal GCG, Wolff LDG, Gonçalves LS, Peres AM, Oliveira FAM. Práticas de atenção perinatal em maternidades de risco habitual: Avaliação na perspectiva de mulheres. Cogitare Enferm. 2016 [citado em 7 out 2018]; 21(2):1-8. Disponível em: https://revistas.ufpr.br/cogitare/article/view/444 $\underline{26}$

22. Gizzo S, Gangi SD, Noventa M, Bacile V, Zambom A, Nardelli GB. Women's choice of positions during labour: Return to the past or a modern way to give birth? A cohort study in Italy. J Biomed Biotechnol. 2014;2014:1-7. DOI: 10.1155/ 2014/638093

23. Sampaio ARR, Bousquat $A$, Barros $C$. Contato pele a pele ao nascer: Um desafio para a promoção do aleitamento materno em maternidade pública no Nordeste brasileiro com o título de Hospital Amigo da Criança. Epidemiol Serv Saúde 2016;25(2):281-90. DOI: 10.5123/ s1679-49742016000200007

24. Carvalho AMVGO, Zangão MOB. Contributo do contacto pele-a-pele na temperatura do recém-nascido. Rev Assoc Port Enferm Obst 2014 [citado em 7 out 2018]; 14:63-7. Disponível em: https://dspace.uevora.pt/rdpc/bitstream/10174/ 13591/1/APEO-2014-Contributo-do-contactopele-a-pele-na-temperatura.pdf 25. Esteves TMB, Daumas RP, Oliveira MIC, Andrade CAF, Leite IC. Fatores associados à amamentação na primeira hora de vida: Revisão sistemática. Rev Saúde Pública 2014;48(4):697703. DOI: $\underline{10.1590 / S 0034-8910.2014048005278}$

Note: Artigo extraído do Trabalho de Conclusão de Curso (Graduação em Enfermagem) intitulado "Práticas Humanizadas em Assistência Parturiente do Hospital de Clínicas de Uberlândia" da Universidade Federal de Uberlândia (UFU).

Recebido em: 26/04/2018

Aprovado em: 01/10/2018

Endereço de correspondência:

Rejane Sousa Romão

Rua Nordau Gonçalves Melo - no 588, ap 101 - Santa

Mônica

CEP: 38408-218 - Uberlândia/MG - Brasil

E-mail: rejaneromao5@hotmail.com 